\title{
Publisher Correction: Ecological and evolutionary approaches to managing honeybee disease
}

Berry J. Brosi, Keith S. Delaplane, Michael Boots and Jacobus C. Roode

Correction to: Nature Ecology \& Evolution 1, 1250-1262 (2017); published online 22 August 2017.

In the HTML version of this Review originally published, a technical error led to the images in Box 2 being swapped over. This was corrected on 28 August 2017.

Published online: 4 December 2017

https://doi.org/10.1038/s41559-017-0394-1 